\title{
Does deviation from a balanced time perspective meaningfully relate to alcohol use and symptoms of anxiety and depression? Comparing results using DBTP and DBTP-r
}

\author{
Michael T. McKay ${ }^{1} \cdot$ Jon C. Cole ${ }^{2}$ \\ Accepted: 5 April 2021 / Published online: 24 April 2021 \\ (C) The Author(s) 2021
}

\begin{abstract}
Time perspective research examines the way in which thoughts and/or feelings about the past, present, and future influence behavior, and deviation from a balanced time perspective (DBTP) has been suggested to be functionally disadvantageous. Recently a revised formula (DBTP-r) was suggested for the derivation of DBTP scores. The present study examined the relationship between self-reported alcohol use and both symptoms of anxiety and depression, with scores on the DBTP and the DBTP-r. Participants $(N=940,48.09 \%$ Male) were recruited as part of a University project and completed the Alcohol Use Disorders Identification Test, the Hospital Anxiety and Depression Scale, and the Zimbardo Time Perspective Inventory. In analyses adjusted for age and sex, DBTP and DBTP-r performed similarly in relation to mental health symptomatology, while only DBTP-r was significantly related to alcohol use. In more adjusted models, more variance was explained in DBTP-r models although neither DBTP score was significantly related to either alcohol use or symptomatology scores when they were operationalised categorically. DBTP-r appears to discriminate better than DBTP, with the caveat that this is the first study to compare them.
\end{abstract}

Keywords Zimbardo Time Perspective Inventory $\cdot$ AUDIT $\cdot$ HADS $\cdot$ Deviation from a balanced time perspective $\cdot$ DBTP

In the broadest sense, temporal psychology refers to the way in which thoughts about and/or feelings towards the past, present and future, influence behavior. A growing body of research continues to suggest that time perspective is significantly and meaningfully (in terms of effect size) related to both mental health and addictive behaviors (e.g., Cole et al., 2016; Kaya Lefèvre et al., 2019; Oyandel \& Buela-Casal, 2014). One of the most widely-used assessment measures of time perspective is the Zimbardo Time Perspective Inventory (ZTPI; Zimbardo \& Boyd, 1999) which assesses cognitive, affective, and behavioral engagement with the past, present, and future, in five domains: past positive (PP), past negative $(\mathrm{PN})$, present hedonistic $(\mathrm{PH})$, present fatalistic $(\mathrm{PF})$, and future $(\mathrm{F})$.

In their development of the ZTPI, Zimbardo and Boyd (1999) reported significant and meaningful correlations

Michael T. McKay

michaelmckay@rcsi.com

1 Department of Psychology, Royal College of Surgeons, Beaux Lane House, Mercer Street, Dublin Dublin 2, Ireland

2 University of Liverpool, Liverpool, UK between depressive symptoms and PN $(r=.59)$, PF $(r=.37)$, PH $(r=-.20)$ and F $(r=-.19)$ time perspectives. Further, these authors reported significant and meaningful relationships between trait anxiety scores and scores on PN $(r=.62)$, PF $(r=.38)$, and PP $(r=-.25)$. More recently, greater PN scores $(d=1.04)$, and lower PH scores $(d=0.87)$ have been reported by patients diagnosed with depression compared to controls (Kaya Lefèvre et al., 2019), while elsewhere, increased PF scores (positively), and increased F time perspective scores (negatively) have been shown to be correlated with allostatic load (Bourdon et al., 2020). In terms of alcohol use, a growing literature has demonstrated that time perspective is also a variable of consequence such that, broadly speaking, a greater present focus or perspective, and/or a reduced future focus or perspective, is significantly related to more problematic drinking behavior (e.g., Barnett et al., 2013; Daugherty \& Brase, 2010; McKay et al., 2018).

Although they did not empirically test it, Zimbardo and Boyd suggested that a balanced time perspective is optimal in terms of human functioning. A balanced time perspective was hypothesized by Zimbardo and Boyd to be represented by relatively high scores on $\mathrm{PP}, \mathrm{PH}$, and $\mathrm{Fe}$, with relatively low scores on PN and PF. Building on this hypothesis, Stolarski 
et al. (2011) developed an equation for the assessment of deviation from a balanced time perspective (DBTP). In order to determine 'balanced' these authors proposed 'ideal' mean scores based on Zimbardo and Boyd's (2008) cross-cultural collective database as follows: $M_{\mathrm{PP}}=4.6, M_{\mathrm{PH}}=3.9, M_{\mathrm{F}}=$ $4.0, M_{\mathrm{PN}}=1.95$, and $\mathrm{M}_{\mathrm{PF}}=1.5$. These scores in turn formed part of an equation: DBTP $=\sqrt{ }\left({ }_{\mathrm{o}} \mathrm{PP}-{ }_{\mathrm{e}} \mathrm{PP}\right)^{2}+\left({ }_{\mathrm{o}} \mathrm{PN}-{ }_{\mathrm{e}} \mathrm{PN}\right)^{2}+$

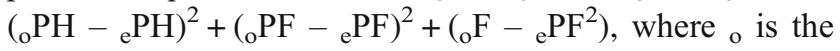
optimal or 'ideal' mean score, and ${ }_{e}$ is the empirical, or observed mean score.

Indeed, the degree of DBTP has been shown to be significantly related to a wide range of variables, in particular wellbeing, mental health, personality, cognitive functioning, self-control, and interpersonal relations (see Stolarski et al., 2020 for a review). In particular, in their systematic review of the DBTP literature, Stolarski et al. (2020) reported on a number of studies which had reported small-to-moderate effect sizes in the relationship between DBTP and alcohol use, as well as studies wherein DBTP scores had been shown to relate meaningfully to mental health and well-being scores. Accordingly, the further the DBTP score is from 'ideal', the lower individuals have been found to score on measures of well-being.

More recently, Jankowski et al. (2020) suggested and empirically tested a different set of 'ideal' means in their development of the DBTP-r, as follows: $M_{\mathrm{PP}}=5.0, M_{\mathrm{PH}}=3.40$, $M_{\mathrm{F}}=5.00, M_{\mathrm{PN}}=1.00$, and $\mathrm{M}_{\mathrm{PF}}=1.00$. In many respects this makes intuitive sense, as otherwise a mean score of $\mathrm{PN}=1.95$ would be considered more adaptive than $\mathrm{PN}=1.00$, suggesting that some degree of PN is better than none. These authors suggested that DBTP scores using this new formula would better discriminate between outcomes than the one previously suggested. In their exploratory study, Jankowski et al. (2020) reported a stronger (higher correlation coefficients) relationship between DBTP-r and life satisfaction (negatively), symptoms of depression, and negative affect (negatively) than was the case for DBTP. Indeed, DBTP was more strongly related to positive affect than DBTP-r (negatively). In large part this is attributable to the fact that the DBTP-r creates a more 'extreme' version of a balanced time perspective. For example, where previously (in the DBTP formula) a mean score for past negative of 1.95 was considered adaptive, the DBTP-r posits that a mean score of 1 (in other words a total absence of past negative thoughts or feelings) is adaptive. The same is true for $\mathrm{F}$ time perspective, where previously a mean of 4 was considered adaptive, whereas in the DBTP-r, a mean of 5 (complete agreement with questions on future thinking or feelings) is considered adaptive. Given the novelty of the DBTP-r approach, no studies have yet examined how scores on that relate to alcohol use.

The present study sought to examine the degree of similarity between results using these formulae, with regard to alcohol use, and symptoms of depression and anxiety. In line with the suggestion and findings of Jankowski et al. (2020), and given the fact that the revised DBTP-r essentially assesses deviation from the most ideal possible circumstance (no PN or PF, and the best possible F and PP scores), we hypothesized to find results using the DBTP-r would be larger in magnitude than those using the DBTP. To the best of our knowledge this is the first study to do so.

\section{Methods}

\section{Participants}

Participants were 940 adults, recruited through a University undergraduate project in a University in the North West of England (aged 17-75 $\left[M_{\text {age }}=22.44, S D=7.36\right] ; N=452$ Males [48.09\%]). The study formed part of a student project, and recruitment to the study was undertaken by University students using opportunistic and snowball sampling of peers, friends, and family members. The study was given ethical approval by the relevant university ethics committee and all participants gave written informed consent.

\section{Measures}

The Alcohol Use Disorders Identification Test (AUDIT) (Saunders et al., 1993) assesses alcohol use. Eight of the items are responded to on a 5-point scale (e.g., "How often do you have six or more drinks on one occasion?") and two on a 3point scale (e.g., "Have you or someone else been injured as a result of your drinking?"). The AUDIT demonstrated good sensitivity (.94) and specificity (.92) in undergraduates (Adewuya, 2005). The original guidelines recommended by Saunders et al. (1993) are used here. Accordingly, a score of eight or more (out of 40) indicates hazardous to harmful alcohol use. Reliability for AUDIT scores in the present study was $\alpha=.83$.

The Hospital Anxiety and Depression Scale (HADS; Zigmond \& Snaith, 1983) was used to screen for symptoms of depression (e.g., "I still enjoy the things I used to enjoy"), and anxiety (e.g., "I get sudden feelings of panic"). The HADS produces scores for anxiety (HADS-A) and depression (HADS-D) on separate subscales that range from zero to twenty-eight. Bjelland et al. (2002) suggested that a score of eight or more on each subscale indicates caseness. Scores on both subscales of the HADS have been shown to be valid and internally consistent (HADS-A: $\alpha=.83$; HADS-D: $\alpha=.82$ ), with equal levels of sensitivity (.80) and specificity (.80; see Bjelland et al., 2002). Both subscales of the HADS demonstrated good internal consistency in the present study (HADSA: $\alpha=0.82$; HADS-D: $\alpha=0.80$ ).

The 56-item Zimbardo Time Perspective Inventory (ZTPI; Zimbardo \& Boyd, 1999) assesses individual differences on 
five subscales. PN ( $\alpha=.82)$ and PP $(\alpha=.80)$, measure a negative and positive view of the past, respectively. $\mathrm{PH}(\alpha=.79)$, measures a risk-taking or impulsive approach to life, oriented toward present pleasure, while PF $(\alpha=.74)$ assesses a pessimistic view of life. Finally, F $(\alpha=.77)$ assesses preparation for the future and orientation to longer-term outcomes. Responses to each item were on a 5-point Likert scale: $1=$ very uncharacteristic of me, through $5=$ very characteristic of me. Reliabilities for ZTPI subscale scores in the present study were as follows: PN, $\alpha=.81$; PP, $\alpha=.70 ; \mathrm{PH}, \alpha=.81$; PF, $\alpha=.74 ; \mathrm{F}, \alpha=.75$. Data were also gathered on participants' age and sex.

\section{Analyses}

Two sets of optimal mean scores were used in order to derive the DBTP and DBTP-r values. In the first place, those formerly suggested on Zimbardo and Boyd's database (www.thetimeparadox. com/surveys, 17-09-2012; the most recent version), were used as follows: $M_{\mathrm{PP}}=3.67 ; M_{\mathrm{PN}}=2.10 ; M_{\mathrm{PH}}=4.33 ; M_{\mathrm{PF}}=1.67 ; M_{\mathrm{F}}=$ 3.69). Secondly, the optimal means from the DBTP-r equation proposed by Jankowski et al. (2020) were also used as follows: $\left.M_{\mathrm{PP}}=5 ; M_{\mathrm{PN}}=1 ; M_{\mathrm{PH}}=3.40 ; M_{\mathrm{PF}}=1 ; M_{\mathrm{F}}=5\right)$. Jankowski et al. reported inconsistencies both in their results, and in the extant literature, for $\mathrm{PH}$ scores, such that, in some cases higher scores were significantly associated with better outcomes, while elsewhere, worse outcomes. Accordingly, a 'neutral' value of 3.4 was hypothesized as optimal or ideal for balance.

Hierarchical regression models (adjusted for sex and age) were computed independently to examine the relationship between DBTP and separately, DBTP-r scores, and AUDIT and HADS scores. A second series of models were then computed which also additionally adjusted for scores on the five ZTPI dimensions, additional to the DBTP or DBTP-r score. Finally, binary logistic models were computed wherein AUDIT and HADS cut-offs (value $\geq 8=$ caseness) were employed in order to examine the relationship between DBTP and DBTP-r with AUDIT and HADS categories. We employed Ferguson's (2009) effect size recommendations as a guide to interpreting results, where $\beta>.20$ is regarded as the minimum for 'practical significance'.

\section{Results}

Given the reliance on an equation that includes empirical or observed mean scores, we only proceeded with further analyses on the basis that Cronbach's alpha estimates for all five ZTPI dimensions were $>.70$. Table 1 displays descriptive statistics, and the results of Pearson's correlations between scores on study measures. Of note, the correlation between the two DBTP scores suggests related but discrete values. However, the two DBTP values correlated with HADS scores to a very similar degree, differing though in the way that they related to AUDIT score. There was no observed significant sex difference between scores on DBTP $\left(M_{\text {Male }}=2.00\right.$ [95\% CI 1.95, $2.05] ; M_{\text {Female }}=1.98$ [95\% CI $\left.=1.94,2.03\right]$ ), nor DBTP-r $\left(M_{\text {Male }}=3.58[95 \%\right.$ CI $3.51,3.64] ; M_{\text {Female }}=3.47[95 \%$ $\mathrm{CI}=3.40,3.54])$. In order to test the degree to which raw DBTP and DBTP-r scores respectively related to scores on HADS-A, HADS-D, and AUDIT, we empirically tested the magnitude of these independent correlation coefficients (Preacher, 2002). Results showed that the difference in magnitude of the DBTP/DBTP-r relationship with HADS-A was not significant $(p=.634)$, nor was the

Table 1 Bivariate Pearson's correlations (two-tailed) between study measures

\begin{tabular}{|c|c|c|c|c|c|c|c|c|c|c|}
\hline & 1 & 2 & 3 & 4 & 5 & 6 & 7 & 8 & 9 & 10 \\
\hline 1 Past positive & - & & & & & & & & & \\
\hline 2 Past negative & -0.32 & - & & & & & & & & \\
\hline 3 Present hedonistic & 0.19 & 0.13 & - & & & & & & & \\
\hline 4 Present fatalistic & 0.18 & 0.23 & 0.37 & - & & & & & & \\
\hline 5 Future & 0.06 & 0.01 & -0.30 & -0.34 & - & & & & & \\
\hline 6 DBTP & 0.04 & 0.33 & -0.14 & 0.56 & -0.26 & - & & & & \\
\hline 7 DBTP-r & -0.03 & 0.33 & 0.25 & 0.63 & -0.56 & 0.79 & - & & & \\
\hline 8 HADS-A & -0.08 & 0.24 & -0.06 & 0.11 & -0.02 & 0.29 & 0.31 & - & & \\
\hline 9 HADS-D & -0.14 & 0.15 & -0.11 & 0.12 & -0.18 & 0.31 & 0.33 & 0.52 & - & \\
\hline 10 AUDIT & 0.12 & 0.00 & 0.33 & 0.11 & -0.26 & -0.03 & 0.16 & 0.07 & 0.02 & - \\
\hline Mean (SD) & $3.36(.66)$ & $3.04(.72)$ & $3.42(.49)$ & $2.62(.61)$ & $3.30(.53)$ & $1.99(.55)$ & $3.52(.73)$ & $5.52(3.97)$ & $2.92(3.01)$ & $11.98(6.22)$ \\
\hline Skewness & -.32 & -.03 & .04 & .07 & -.09 & .42 & -.03 & .63 & 1.22 & .46 \\
\hline Kurtosis & 2.94 & 2.49 & 3.03 & 2.84 & 3.10 & 3.03 & 2.87 & 2.76 & 4.05 & 3.13 \\
\hline
\end{tabular}

$D B T P$, Deviation from a Balanced Time Perspective $(-\mathrm{r}=$ revised); HADS, Hospital Anxiety and Depression Scale $(-\mathrm{A}=\mathrm{Anxiety},-\mathrm{D}=\mathrm{Depression})$; AUDIT, Alcohol Use Disorders Identification Test. Italicized coefficients $=p<.01$ 
difference in magnitude of the relationships with HADS-D $(p=.630)$. However, DBTP-r was correlated with AUDIT score to a significantly greater degree than DBTP $(p<.001)$.

Table 2 displays the results of hierarchical regression models examining the relationship between DBTP versions, and scores on criterion variables, adjusted for age and sex. The total variance explained by the DBTP-r models was a little higher in all cases than that explained by the corresponding DBTP model. DBTP score was not significantly related to AUDIT score, whereas DBTP-r score was, however, not to Ferguson's 'practically significant' $(\beta \geq .20)$ threshold. In both models, higher AUDIT score was significantly related to being younger, and being male. Higher HADS-A score was significantly related to a greater DBTP in both models (DBTP and DBTP-r), with the $\beta$ values both 'practically significant' (Ferguson, 2009). Further, higher HADS-A score was also significantly related to being female $(p<.05)$, but only in the DBTP-r model, and with a small effect size. Higher HADS-D score was significantly related to a greater DBTP in both models, with practically significant effect sizes. Additionally, higher HADS-D score was significantly related to being older, in both DBTP models.

In order to further examine these relationships, we re-ran the models adjusting for ZTPI dimension scores. The full output for these models is displayed in Supplementary S1. Results are displayed in Table 3, and show that more variance was explained in these models than that explained in those including $\operatorname{DBTP}(-r)$ scores only (Table 2). Adjusting for ZTPI dimension scores, both DBTP models remained significantly associated with HADS-A and HADS-D scores, and largely to a practically significant degree (beta values). Of note, AUDIT scores in both models became non-significant with the inclusion of dimension scores.

Because cut-offs have been established for both the AUDIT and the HADS, we further explored the relationship between DBTP, DBTP-r, and these variables using scale categories. In both cases a score $\geq 8$ is considered to be indicative of problematic alcohol use, and problematic level of symptomatology. In all six models (detailed in Supplementary S2 and adjusted for age and sex), neither DBTP nor DBTP-r scores were significantly related to membership of AUDIT or HADS categories.

\section{Discussion}

Comparing raw correlational results, and results for both the HADS-A and HADS-D in models adjusted only for sex and age, seems to suggest that the DBTP and DBTP-r function quite similarly, with the caveat that the DBTP-r model explained slightly more overall variance. Results for the AUDIT, however, illustrate that DBTP-r scores discriminated drinking behaviour in a way that the DBTP did not, although the effect size was small. In their literature review of the DBTP, Stolarski et al. (2020) illustrated that DBTP relates to criterion variables to different degrees, and not well overall to alcohol use.

Results in models adjusted for ZTPI dimensions illustrate that for HADS-A and HADS-D, DBTP-r was better at discriminating than the DBTP, with larger standardized beta values and overall amount of variance explained. However, it is somewhat disappointing that neither the DBTP nor DBTP-r was significantly associated with more problematic
Table 2 Results of hierarchical regression models examining the relationship between both DBTP and DBTP-r and criterion variables

\begin{tabular}{|c|c|c|c|c|c|c|c|c|c|c|}
\hline & \multicolumn{5}{|c|}{$\underline{\text { DBTP models }}$} & \multicolumn{5}{|c|}{ DBTP-r models } \\
\hline & B & SE & $\beta$ & $p$ value & $\mathrm{R}^{2}$ & $\mathrm{~B}$ & SE & $\beta$ & $p$ value & $\mathrm{R}^{2}$ \\
\hline AUDIT & & & & & 0.06 & & & & & 0.09 \\
\hline $\mathrm{DBTP} / \mathrm{r}$ & -0.09 & 0.36 & -0.01 & .809 & & 1.41 & 0.27 & 0.17 & $<.001$ & \\
\hline Age & -0.19 & 0.03 & -0.22 & $<.001$ & & -0.20 & 0.03 & -0.23 & $<.001$ & \\
\hline Sex & -1.48 & 0.39 & -0.12 & $<.001$ & & -1.32 & 0.39 & -0.11 & .001 & \\
\hline HADS-A & & & & & 0.08 & & & & & 0.10 \\
\hline $\mathrm{DBTP} / \mathrm{r}$ & 2.17 & 0.23 & 0.30 & $<.001$ & & 1.73 & 0.17 & 0.32 & $<.001$ & \\
\hline Age & -0.03 & 0.02 & -0.05 & .084 & & -0.02 & 0.02 & -0.04 & .208 & \\
\hline Sex & 0.42 & 0.25 & 0.05 & .088 & & 0.58 & 0.25 & 0.07 & .020 & \\
\hline HADS-D & & & & & 0.11 & & & & & 0.12 \\
\hline $\mathrm{DBTP} / \mathrm{r}$ & 1.65 & 0.17 & 0.30 & $<.001$ & & 1.34 & 0.13 & 0.33 & $<.001$ & \\
\hline Age & 0.04 & 0.01 & 0.11 & .001 & & 0.05 & 0.01 & 0.12 & $<.001$ & \\
\hline Sex & -0.04 & 0.19 & -0.01 & .811 & & 0.07 & 0.18 & 0.01 & .719 & \\
\hline
\end{tabular}

$D B T P$, Deviation from a Balanced Time Perspective $(-\mathrm{r}=$ revised); HADS, Hospital Anxiety and Depression Scale $(-\mathrm{A}=$ Anxiety, $-\mathrm{D}=$ Depression $)$; AUDIT, Alcohol Use Disorders Identification Test. $\mathrm{R}^{2}$ values are adjusted $\mathrm{R}^{2}$ 
Table 3 Results of hierarchical regression models examining the relationship between both DBTP and DBTP-r and criterion variables. Results are adjusted for ZTPI dimension mean scores

\begin{tabular}{|c|c|c|c|c|c|c|c|c|c|c|}
\hline & \multicolumn{5}{|c|}{ DBTP Models } & \multicolumn{5}{|c|}{ DBTP-r Models } \\
\hline & B & SE & $\beta$ & $p$ value & $\mathrm{R}^{2}$ & B & SE & $\beta$ & $p$ value & $\mathrm{R}^{2}$ \\
\hline AUDIT & & & & & 0.18 & & & & & 0.18 \\
\hline $\mathrm{DBTP} / \mathrm{r}$ & -0.39 & 0.50 & -0.03 & .432 & & 0.20 & 0.39 & 0.02 & .609 & \\
\hline Age & -0.15 & 0.03 & -0.17 & $<.001$ & & -0.15 & 0.03 & -0.17 & $<.001$ & \\
\hline Sex & -1.25 & 0.37 & -0.10 & .001 & & -1.24 & 0.37 & -0.10 & .001 & \\
\hline HADS-A & & & & & 0.12 & & & & & 0.17 \\
\hline $\mathrm{DBTP} / \mathrm{r}$ & 2.16 & 0.33 & 0.30 & $<.001$ & & 2.54 & 0.25 & 0.47 & $<.001$ & \\
\hline Age & -0.04 & 0.02 & -0.08 & .018 & & -0.04 & 0.02 & -0.07 & .025 & \\
\hline Sex & 0.42 & 0.24 & 0.05 & .086 & & 0.56 & 0.24 & 0.07 & .018 & \\
\hline HADS-D & & & & & 0.14 & & & & & 0.17 \\
\hline $\mathrm{DBTP} / \mathrm{r}$ & 1.55 & 0.25 & 0.28 & $<.001$ & & 1.61 & 0.19 & 0.39 & $<.001$ & \\
\hline Age & 0.04 & 0.01 & 0.09 & .005 & & 0.04 & 0.01 & 0.09 & .003 & \\
\hline Sex & -0.02 & 0.18 & -0.00 & .906 & & 0.06 & 0.18 & 0.01 & .730 & \\
\hline
\end{tabular}

ZTPI, Zimbardo Time Perspective Inventory; DBTP, Deviation from a Balanced Time Perspective $(-\mathrm{r}=$ revised); $H A D S$, Hospital Anxiety and Depression Scale $(-\mathrm{A}=$ Anxiety, $-\mathrm{D}=$ Depression); AUDIT, Alcohol Use Disorders Identification Test alcohol use or symptomatology in models examining AUDIT and HADS scores categorically. It would have spoken more to the explanatory power of the DBTP, and in particular to the revised DBTP-r had scores been able to differentiate nonproblematic from potentially problematic behaviour.

The results of the present study need to be understood and interpreted in the context of a broader, ongoing debate about the ZTPI, and the use of both the idea of a balanced time perspective and the associated DBTP. In the first instance, the psychometric validity and internal consistency of ZTPI scores continues to be questioned (Davis \& Cernas-Ortiz, 2017; Worrell et al., 2018), with multiple sample-specific, shortened versions created in order to achieve acceptable reliability and fit (e.g., Kocayoruk \& Simsek, 2020; Sircova et al., 2014; Temple et al., 2019). The internal consistency estimates for ZTPI domains in the present study were all satisfactory, however, McKay and Worrell (2020) have highlighted the fact that internal consistency and/or psychometric validity of ZTPI scores were problematic in many of the manuscripts retained for Stolarski et al. (2020) review.

Following on from this, the temporal psychology field needs to reconsider a previously asked question (McKay et al., 2019), namely, the degree to which both the idea of a balanced time perspective and by extension, deviation from a balanced time perspective, are mere exercises in empiricism. Previous manuscripts have drawn attention to the fact that while Zimbardo and Boyd's balanced time perspective idea is intuitive, it rarely emerges in, for example, cluster analyses (e.g. McKay et al., 2019). Relatedly, it appears self-evident that an individual reporting no $\mathrm{PN}$, and the highest possible PP score (part of the metrics used in the DBTP-r) would be highly functional. However, in reality, for whom is such a situation representative? It is unlikely that any such person would exist, therefore, if the DBTP/DBTP-r are merely measuring deviation from an unrealistic psychological reality, it is difficult to know how either advances our understanding of human (temporal) psychology greatly.

In conclusion, the DBTP-r did explain variance in drinking behaviour that DBTP scores did not. This is not altogether surprising as, the DBTP-r is essentially an extreme version of the DBTP. By this we mean that essentially optimal mean score on the functional ZTPI dimensions (PP and F) has been pushed to the extreme (mean $=5.00$ ), with the opposite true for the dysfunctional ZTPI dimensions (PN and PF). This is the first study to compare scores using the DBTP with those using the DBTP-r, and results must be interpreted in light of that limitation. In other words, it would not be appropriate to make definitive claims on the basis of results from a single follow-up study. Further studies will be required in order to determine whether these findings are both generalizable and replicable.

Supplementary Information The online version contains supplementary material available at https://doi.org/10.1007/s12144-021-01719-2.

Funding Open Access funding provided by the IReL Consortium. There was no funding associated with this study.

Data Availability The datasets generated during and/or analysed during the current study are not publicly available as agreed with participants, but are available from the corresponding author on reasonable request.

\section{Declarations}

Ethics Approval This study received ethical approval from the University of Liverpool Ethics Committee: IPHS 1415-131 and IPHS 1415-131.1. 
All procedures performed in studies involving human participants were in accordance with the ethical standards of the University of Liverpool and with the 1964 Helsinki Declaration and its later amendments or comparable ethical standards.

Informed Consent Informed consent was obtained from all participants included in the study.

Conflict of Interest On behalf of all authors, the corresponding author states that there is no conflict of interest.

Open Access This article is licensed under a Creative Commons Attribution 4.0 International License, which permits use, sharing, adaptation, distribution and reproduction in any medium or format, as long as you give appropriate credit to the original author(s) and the source, provide a link to the Creative Commons licence, and indicate if changes were made. The images or other third party material in this article are included in the article's Creative Commons licence, unless indicated otherwise in a credit line to the material. If material is not included in the article's Creative Commons licence and your intended use is not permitted by statutory regulation or exceeds the permitted use, you will need to obtain permission directly from the copyright holder. To view a copy of this licence, visit http://creativecommons.org/licenses/by/4.0/.

\section{References}

Adewuya, A. O. (2005). Validation of the alcohol use disorders identification test (Audit) as a screening tool for alcohol-related problems among Nigerian university students. Alcohol and Alcoholism, 40(6), 575-577.

Barnett, E., Spruijt-Metz, D., Unger, J. B., Rohrbach, L. A., Sun, P., \& Sussman, S. (2013). Bidirectional associations between future time perspective and substance use among continuation high-school students. Substance Use and Misuse, 48, 574-580.

Bjelland, I., Dahl, A. A., Haug, T. T., \& Neckelmann, D. (2002). The validity of the hospital anxiety and depression scale; an updated review. Journal of Psychosomatic Research, 52(2), 69-77.

Bourdon, O., Raymond, C., Martin, M-F., Olivera-Figueroa, L., Lupien, S. J., \& Juster, R-P. (2020). A time to be chronically stressed? Maladaptive time perspectives are associated with allostatic load. Biological Psychology, 152, 107871.

Cole, J.C., Andretta, J.R., \& McKay, M.T. (2016). The relationship between temporal profiles and alcohol-related problems in University Undergraduates: Results from the United Kingdom. Addictive Behaviors, 55, 15-18.

Daugherty, J. R., \& Brase, G. L. (2010). Taking time to be healthy: Predicting health behaviors with delay discounting and time perspective. Personality and Individual Differences, 48, 202-207.

Davis, M. A., \& Cernas-Ortiz, D. A. (2017). Revisiting the structural and nomological validity of the Zimbardo time perspective inventory. Personality and Individual Differences, 104, 98-103.

Ferguson, C. J. (2009). An effect size primer: A guide for clinicians and researchers. Professional Psychology: Research and Practice, 40, $532-538$.

Jankowski, K. S., Zajenkowski, M., \& Stolarski, M. (2020). What are the optimal levels of time perspectives? Deviation from the balanced time perspective-revisited (DBTP-r). Psychologica Belgica, 60, 164-183.

Kaya Lefèvre, H., Mirabel-Sarron, C., Docteur, A., Leclerc, V., Laszcz, A., Gorwood, P., \& Bungener, C. (2019). Time perspective differences between depressed patients and non-depressed participants, and their relationships with depressive and anxiety symptoms. Journal of Affective Disorders, 246, 320-326.

Kocayoruk, E., \& Simsek, O. F. (2020). The validity and reliability of the Turkish brief version of the Zimbardo time perspective inventory. Dusunen Adam, 33, 40-49.

McKay, M.T., Perry, J.L., Cole, J.C., \& Worrell, F.C. (2018). What time is it? Temporal psychology measures relate differently to alcoholrelated health outcomes. Addiction Research and Theory, 26, 20-27.

McKay, M.T., Worrell, F.C., Zivkovic, U., Temple, E., Mello, Z.R., Musil,. B., Cole, J.C., Andretta, J.R., \& Perry, J.L. (2019). A Balanced Time Perspective: Is it an exercise in empiricism, and does it relate meaningfully to Health and Well-being outcomes? International Journal of Psychology, 54, 775-785.

McKay, M.T., \& Worrell, F.C. (2020). Technical Comment on Stolarski, M., Zajenkowski, M., Jankowski, K. S., \& Szymaniak, K. (2020). Deviation from the balanced time perspective: A systematic review of empirical relationships with psychological variables. Personality and Individual Differences, 156, 109772. Personality and Individual Differences, 165, 110132.

Oyandel, C., \& Buela-Casal, G. (2014). Time perception and psychopathology: Influence of time perspective on quality of life of severe mental illness. Actas Espanolas de Psiquiatria, 42(3), 99-107.

Preacher, K. J. (2002). Calculation for the test of the difference between two independent correlation coefficients [Computer software]. Available from http://quantpsy.org.

Saunders, J. B., Aasland, O. G., Babor, T. F., dela Fuente, J. R., \& Grant, M. (1993). Development of the alcohol use disorders identification test (AUDIT): WHO collaborative project on early detection of persons with harmful alcohol consumption-II. Addiction, 88, 791-804.

Sircova, A., van de Vijver, F. J. R., Osin, E., Milfont, T. L., Fieulaine, N., Kislali-Erginbilic, A.,...54 members of the International Time Perspective Research Project (2014). A global look at time: A 24country study of equivalence of the Zimbardo time perspective inventory. SAGE Open, 4(1), 1-12. https://doi.org/10.1177/ 2158244013515686.

Stolarski, M., Bitner, J., \& Zimbardo, P. G. (2011). Time perspective, emotional intelligence and discounting of delayed awards. Time \& Society, 20(3), 346-363.

Stolarski, M., Zajenkowski, M., Jankowski, K. S., \& Szymaniak, K. (2020). Deviation from the balanced time perspective: A systematic review of empirical relationships with psychological variables. Personality and Individual Differences, 156, 109772.

Temple, E., Perry, J.L., Worrell, F.C., Zivkovic, U., Mello, Z.R., Musil, B., Cole, J.C., \& McKay, M.T. (2019). The Zimbardo Time Perspective Inventory: Time for a new strategy, not more new shortened versions. Time and Society, 28, 1167-1180.

Worrell, F.C., McKay, M.T., Cole, J.C., Zivkovic, U., Perry, J.R., Musil, B., \& Mello, Z.R. (2018). A Theoretical Approach to the Zimbardo Time Perspective Inventory: Results from America, Australia, Slovenia, and the United Kingdom. European Journal of Psychological Assessment, 34, 41-51.

Zigmond, A. S., \& Snaith, R. P. (1983). The hospital anxiety and depression scale. Acta Psychiatrica Scandinavica, 67, 361-370.

Zimbardo, P. G., \& Boyd, J. N. (1999). Putting time in perspective: A valid, reliable individual-differences metric. Journal of Personality and Social Psychology, 77, 1271-1288. https://doi.org/10.1037// 0022-3514.77.6.1271.

Zimbardo, P. G., \& Boyd, J. N. (2008). The time paradox: The new psychology of time that will change your life. Free Press.

Publisher's Note Springer Nature remains neutral with regard to jurisdictional claims in published maps and institutional affiliations. 